\title{
Distribution of aquaporin water channels AQP1 and AQP5 in the ductal system of the human pancreas
}

\author{
B Burghardt, M-L Elkjær, T-H Kwon, G Z Rácz, G Varga, M C Steward, S Nielsen
}

Gut 2003;52:1008-1016

\begin{abstract}
Background: The exocrine pancreas secretes large volumes of isotonic fluid, most of which originates from the ductal system. The role of aquaporin (AQP) water channels in this process is unknown. Methods: Expression and localisation of known AQP isoforms was examined in normal human pancreas, pancreatic adenocarcinoma, and pancreatic cell lines of ductal origin (Capan-1, Capan-2, and HPAF) using reverse transcriptase-polymerase chain reaction and immunohistochemistry.

Results: Messenger RNAs for AQP1, $-3,-4,-5$, and -8 were detected in normal pancreas and in pancreatic adenocarcinoma. The cell lines expressed AQP3, -4 , and -5 but lacked AQP1 and AQP8. Immunohistochemistry of normal pancreas revealed that AQP 1 is strongly expressed in centroacinar cells and in both the apical and basolateral domains of intercalated and intralobular duct epithelia. AQP1 expression declined with distance along the small interlobular ducts and was not detectable in larger interlobular ducts. AQP3 and AQP4 were not detectable by immunohistochemistry. AQP5 was observed at the apical membrane of intercalated duct cells and also in duct associated mucoid glands. AQP8 was confined to the apical pole of acinar cells. Both AQP1 and AQP5 were colocalised with cystic fibrosis transmembrane conductance regulator (CFTR) at the apical membrane of intercalated duct cells.

Conclusions: AQP1 and AQP5 are strongly expressed in the intercalated ducts of the human pancreas. Their distribution correlates closely with that of CFTR, a marker of ductal electrolyte secretion. This suggests that fluid secretion is concentrated in the terminal branches of the ductal tree and that both AQP1 and AQP5 may play a significant role.
\end{abstract}

See end of article for authors' affiliations

Correspondence to: Dr S Nielsen, The Wate and Salt Research Center, Aarhus, DK-8000 Aarhus, Denmark; sn@ana.au.dk

Accepted for publication 5 March 2003
T he human pancreas secretes approximately l litre of bicarbonate rich juice into the duodenum every day. Much

of the fluid is thought to be produced by epithelial cells lining the ductal system rather than by acinar cells. ${ }^{1}$ The near isotonicity of pancreatic juice ${ }^{2}$ suggests that transepithelial water movement is coupled osmotically to the active transport of electrolytes. However, such a mechanism requires a high transepithelial water permeability.

Aquaporin (AQP) water channels, first discovered in $1991,{ }^{34}$ are known to enhance the water permeabilities of a wide range of epithelia. The aquaporins are small integral membrane proteins whose six $\alpha$-helical membrane spanning domains surround a highly selective aqueous pore. ${ }^{5-7}$ Currently, at least 10 mammalian members of the family have been identified and many show very specific tissue localisation..$^{8-10}$

Until recently, only AQP8 was thought to be associated with the secretory epithelia of the exocrine pancreas. ${ }^{11}$ However immunohistochemistry of the rat pancreas revealed that this isoform is localised in the acinar cells, which are not thought to be the principal site of fluid secretion. ${ }^{12}$ Although AQPI was known to be present in the vasculature, recent studies have provided evidence that AQPI is also expressed in interlobular ducts. ${ }^{13}$ Low levels of mRNA for AQP5, an isoform associated with fluid secretion in other exocrine glands, ${ }^{14}{ }^{15}$ have also been detected in rat pancreas but protein expression could not be demonstrated by immunohistochemistry. ${ }^{12}{ }^{13}$ The only information on aquaporin expression in the human pancreas is an early immunofluorescence study which claimed to detect AQPl expression in acinar cells. ${ }^{16}$

In order to resolve these conflicting observations, we have used immunohistochemistry in combination with reverse transcriptase-polymerase chain reaction (RT-PCR) to explore expression and localisation of a number of different aquaporin isoforms in the human pancreas, and also in the rat and mouse pancreas for comparison. As the cystic fibrosis transmembrane conductance regulator (CFTR) protein is a key transporter involved in secretin evoked pancreatic electrolyte secretion, ${ }^{1}$ we have used this as a functional marker to identify the ductal segments most likely to be the principal sites of fluid secretion.

\section{METHODS}

\section{Tissue samples}

Samples of human pancreas, pancreatic adenocarcinoma, salivary gland, and brain were taken from adult patients undergoing surgery who had previously given informed consent according to approved local procedures. Tissue samples destined for RT-PCR analysis were frozen in liquid nitrogen and stored at $-70^{\circ} \mathrm{C}$. Samples for immunohistochemistry were immersion fixed in formalin and processed for paraffin embedding.

\section{Cell culture}

Three human pancreatic adenocarcinoma cell lines of ductal origin were obtained from the American Type Culture Collection. Capan- 1 and Capan-2 cells $\mathrm{s}^{17}{ }^{18}$ were maintained in RPMI 1640 medium (Sigma, St Louis, Missouri, USA). HPAF cells ${ }^{19}$ were maintained in Dulbecco's modified Eagle's medium (Sigma). Culture media were supplemented with $10 \%$ fetal calf serum, $100 \mathrm{U} / \mathrm{ml}$ penicillin, and $100 \mu \mathrm{g} / \mathrm{ml}$ streptomycin (Sigma). Cells were routinely cultivated in a humidified atmosphere of $5 \% \mathrm{CO}_{2}$ and $95 \%$ air at $37^{\circ} \mathrm{C}$.

Abbreviations: $A Q P$, aquaporin; CCK, cholecystokinin; CFTR, cystic fibrosis transmembrane conductance regulator; PBS, phosphate buffered saline; RT-PCR, reverse transcriptase-polymerase chain reaction. 
Table 1 Primer sequences used for polymerase chain reactions for aquaporins $A Q P 1,-3,-4,-5$, and -8

\begin{tabular}{|c|c|c|c|}
\hline & Sense & Antisense & Product size (bp) \\
\hline $\mathrm{XS13}$ & 5'-CTGGCTAAGTTGGTTGCTTT-3' & 5'-GCAGCTGATCAAGACTGGA-3' & 500 \\
\hline AQP1 & 5'-GTCTTCATCAGCATCGGTTC-3' & 5'-GTCGGCATCCAGGTCATACT-3' & 701 \\
\hline AQP3 & 5'-CCTTTGGCTTTGCTGTCACTC- $3^{\prime}$ & 5'-ACGGGGTTGTGTAAGGGTCA-3' & 373 \\
\hline AQP4 & 5'-TCCCTTTGCTTTGGACTCAG-3' & $5^{\prime}$-ТTCССCTTCTTСТCСТCTCC- $3^{\prime}$ & 719 \\
\hline AQP5 & 5'-CTTCCTCAAGGCCGTGTTC-3' & 5'-GCTGGAAGGTCAGAATCAGC-3' & 398 \\
\hline AQP8 & 5'-GGTACGAACGGTTTGTGCAG-3' & 5'-CCATCTCCAATGAAGCACCT- $3^{\prime}$ & 667 \\
\hline
\end{tabular}

XS13, acidic ribosomal phosphoprotein used as an internal control.

\section{RNA extraction and RT-PCR}

Total RNA was extracted from human pancreas, pancreatic adenocarcinoma, and pancreatic cell lines using conventional methods ${ }^{20}$ Reverse transcription was carried out on $5 \mu \mathrm{g}$ RNA at $42^{\circ} \mathrm{C}$ using $200 \mathrm{U}$ reverse transcriptase (SuperScript II; Gibco, Grand Island, New York, USA) according to the manufacturer's instructions.

The PCR reactions for AQP1, $-3,-4$, and -8 were performed in a final volume of $20 \mu \mathrm{l}$ using $0.5 \mathrm{U}$ Takara Taq polymerase (Takara, Shiga, Japan), $0.5 \mu \mathrm{M}$ each of the primers (table 1), and $200 \mu \mathrm{M}$ dNTP in $10 \mathrm{mM}$ Tris $\mathrm{HCl}$ buffer (pH 8.3) containing $50 \mathrm{mM} \mathrm{KCl}$ and $1.5 \mathrm{mM} \mathrm{MgCl}_{2}$. For AQP5, $0.5 \mathrm{U}$ Promega Taq polymerase (Promega, Madison, Wisconsin, USA) was used together with 0.3 U Pfu DNA polymerase (Promega) in $20 \mathrm{mM}$ Tris $\mathrm{HCl}$ buffer ( $\mathrm{pH} 8.8$ ) containing $10 \mathrm{mM} \mathrm{KCl}, 10 \mathrm{mM}$ $\left(\mathrm{NH}_{4}\right)_{2} \mathrm{SO}_{4}, 2 \mathrm{mM} \mathrm{MgSO}{ }_{4}, 0.1 \%$ Triton X-100, and $0.1 \mathrm{mg} / \mathrm{ml}$ nuclease free bovine serum albumin. After denaturation at $94^{\circ} \mathrm{C}$ for three minutes, 30 cycles of amplification were performed for $\mathrm{AQP1},-4$, and $-8\left(94^{\circ} \mathrm{C}, 30\right.$ seconds; $63^{\circ} \mathrm{C}, 45$ seconds; $72^{\circ} \mathrm{C}, 60$ seconds), for $\mathrm{AQP} 3\left(94^{\circ} \mathrm{C}, 30\right.$ seconds; $65^{\circ} \mathrm{C}$, 45 seconds; $72^{\circ} \mathrm{C}$, 60 seconds), and for AQP5 $\left(94^{\circ} \mathrm{C}, 30\right.$ seconds; $63^{\circ} \mathrm{C}, 30$ seconds; $72^{\circ} \mathrm{C}, 120$ seconds). Amplification was terminated by a final extension step at $72^{\circ} \mathrm{C}$ for 10 minutes in all cases. RT-PCR products were visualised on 1.5\% agarose gels stained with ethidium bromide and were sequenced with the PRISM BigDye Terminator v3.0 sequencing kit (Applied Biosystems, Foster City, California, USA).

\section{Antibodies}

Previously characterised affinity purified polyclonal primary antibodies were used as follows: rabbit antirat/human AQP1 (Alpha Diagnostic International); rabbit antirat AQP3, LL178AP ${ }^{21}$; rabbit antirat AQP4, LL182AP ${ }^{22}$; rabbit antirat AQP5 (Alpha Diagnostic International, San Antonio, Texas, USA); rabbit antihuman AQP5 (kindly provided by Peter Agre and Landon King, Johns Hopkins University Medical School) $)^{23}$; and rabbit antihuman AQP8, 2669AP. For CFTR, a monoclonal mouse antihuman antibody was used (25031; R\&D Systems, Minneapolis, Minnesota, USA). Non-immune IgG and non-immune rabbit serum were obtained from Dako (Glostrup, Denmark).

\section{Immunohistochemistry}

Paraffin embedded blocks of human pancreas were kindly provided by the Departments of Pathology, Aarhus University Hospitals. Non-tumour tissue was obtained from patients with pancreatic carcinoma. As described in detail elsewhere, ${ }^{24}$ $2 \mu \mathrm{m}$ sections were exposed to the primary antibody overnight, then to a horseradish peroxidase conjugated goat antirabbit secondary antibody (P448; Dako), and were finally counterstained with Mayer's haematoxylin. For fluorescence microscopy, labelling was visualised with Alexa Fluor 488 goat antirabbit IgG (Molecular Probes, Leiden, the Netherlands) for AQP1 and -5, and with Alexa Fluor 546 goat antimouse IgG (Molecular Probes) for human CFTR. Sections were examined with a Leica SP2 confocal laser microscope.

Tissue was prepared for electron microscopy by freeze substitution, as described previously. ${ }^{12}$ Immunolabelling was per- formed on ultrathin sections $(60 \mathrm{~nm})$ which were incubated with rabbit antirat AQPI overnight. Labelling was visualised with goat antirabbit IgG conjugated to $10 \mathrm{~nm}$ colloidal gold particles (BioCell Research Laboratories, Cardiff, UK). Control sections were incubated with non-immune IgG at $0.2 \mu \mathrm{g} / \mathrm{ml}$ dilution. Sections were stained with uranyl acetate for 10 minutes and examined on a Phillips CM100 electron microscope.

\section{RESULTS \\ RT-PCR analysis}

To examine aquaporin expression at the mRNA level, PCR primers were designed to amplify cDNA sequences specific to individual human AQP isoforms. The ribosomal phosphoprotein XS13 was used as an internal reference. ${ }^{25}$ PCR products of the expected size were obtained from normal human pancreas using primers specific for AQP1, $-3,-4,-5$, and - 8 (fig 1 ). The nucleotide sequences of the PCR products were all at least $98 \%$ identical with the published human sequences.

Expression levels varied between samples (fig 1). AQP1 mRNA was readily detected in normal pancreas and pancreatic adenocarcinoma but was not present in any of the three cell lines. Messenger RNAs for AQP3 and AQP4 were clearly present in the normal pancreas and in the adenocarcinoma but were expressed at lower levels in the pancreatic cell lines. AQP5 mRNA was expressed at low levels in all of the samples while AQP8 mRNA was detected in normal human pancreas, and in one of the two adenocarcinoma samples, but not in the cell lines.

\section{Immunohistochemical localisation of AQP1}

Immunoperoxidase labelling was performed on paraffin sections of normal human pancreas using an affinity purified primary antibody for AQP1 (fig 2A). Labelling was completely abolished when the primary antibody was pre-adsorbed with the immunising peptide (fig 2B). In fig 2A, clusters of small AQP1 labelled cells and, occasionally, isolated cells can be seen within and among the acini throughout the lobular exocrine tissue. None of the secretory cells within the acini, identifiable by the zymogen granules present at their apical pole, was labelled. However, centroacinar cells, located at the centre of many of the acini, and sometimes extending to the edges of the acini, showed strong labelling of the plasma membranes (fig 2C-F).

Also strongly labelled were the intercalated ducts (fig 2A). These are seen as elongated and occasionally branching groups of cells in longitudinal section (fig 2G) and as cartwheel-like structures in transverse section (fig 2E, F). Both the apical and basolateral plasma membrane domains exhibited AQPI labelling, and there was also some diffuse labelling of the cytoplasm. The abrupt transition from unlabelled acinar cells to strongly labelled intercalated duct cells, shown in fig $2 \mathrm{G}$, is particularly striking.

AQP1 was also detected in capillaries and other small blood vessels but was absent from the larger vessels and also from the endocrine cells in the islets of Langerhans (fig 3B). 

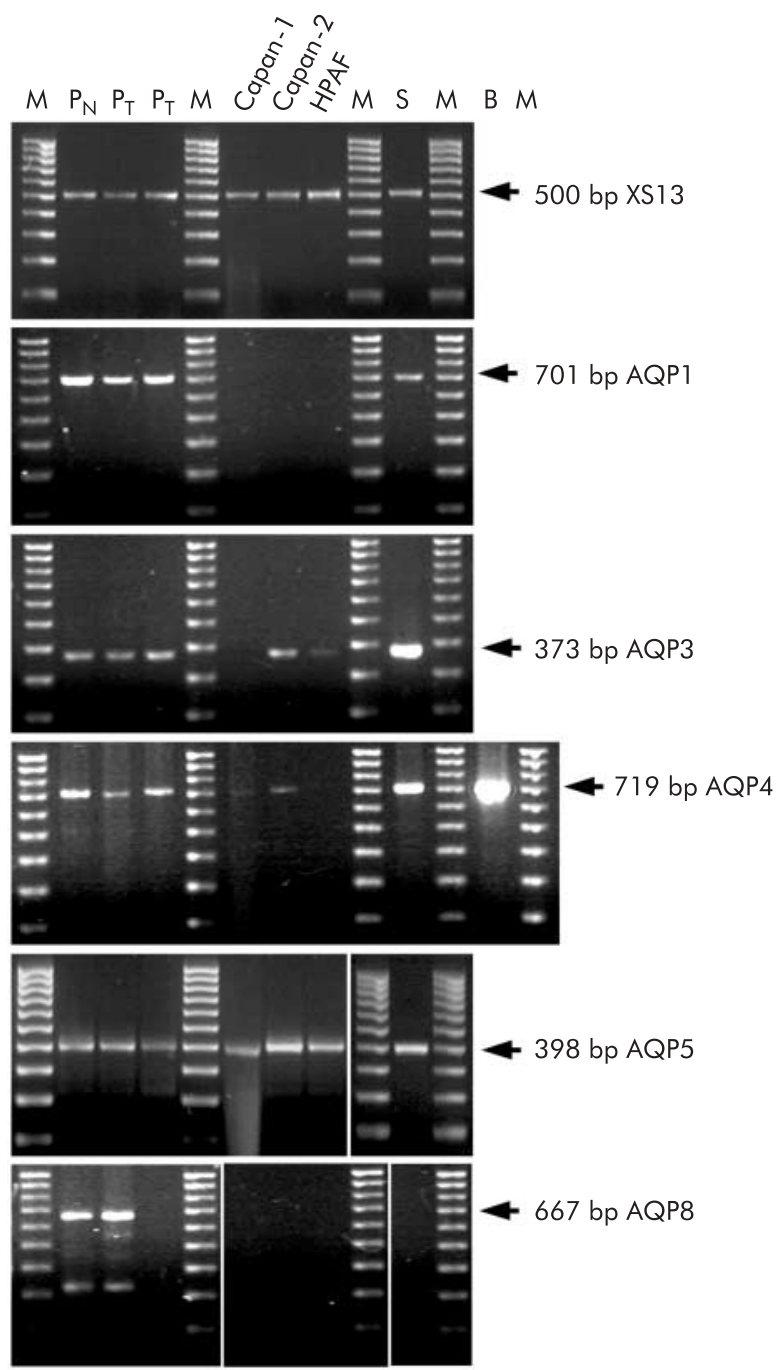

Figure 1 Reverse transcriptase-polymerase chain reaction (RT-PCR) analysis of aquaporin (AQP) expression in human pancreas, pancreatic tumours, and ductal cell lines. Agarose gels stained with ethidium bromide showing PCR products obtained with RNA extracted from human tissues and cell lines using specific primers for XS13 (an acidic ribosomal phosphoprotein used as an internal control), $A Q P 1,-3,-4,-5$, and -8 . The concentration of the pancreatic CDNA was increased threefold for the AQP5 reaction. The arrows indicate the expected sizes of the products based on published sequences. Lane 1, normal pancreas $\left(P_{N}\right)$; lanes 2, 3, pancreatic tumours $\left(\mathrm{P}_{T}\right)$; lane 4, Capan-1 cells; lane 5, Capan-2 cells; lane 6, HPAF cells; lane 7, parotid salivary gland (S); $M$, marker. The additional lane for AQP4 shows the positive control from human hippocampus (B).

\section{Colocalisation of AQP1 and CFTR in intercalated ducts}

To compare localisation of AQP1 with the expected site of fluid secretion in the ductal system, CFTR was chosen as a functional marker of the secretory cells for double label immunofluorescence experiments. Figure $2 \mathrm{H}$ shows localisation of AQPI (green) and this clearly follows the same general pattern as in fig $2 \mathrm{~A}$. The more restricted distribution of CFTR is shown in the same section (red, fig 2I) where it is confined to the apical membrane of the intercalated ducts and also acinar cells. When the two images are merged (fig $2 \mathrm{~J}$ ), it is evident that AQP1 and CFTR are colocalised apically in the intercalated ducts. The one or two points of CFTR labelling that do not coincide with AQP1 labelling in fig $2 \mathrm{~J}$ indicate CFTR expression at the apical membrane of the acinar cells. Extensive regions of AQPI labelling that do not coincide with CFTR labelling, on the other hand, are clearly due to the pres- ence of AQPl in the basolateral membrane domains of the intercalated duct cells where CFTR is absent.

\section{AQP1 distribution in intralobular and interlobular ducts of human pancreas}

Although less common than intercalated ducts, intralobular ducts were occasionally observed between acini within lobules. These were labelled by the AQPI antibody at both the apical and basolateral membranes although less strongly at the latter (fig 3A, 3B). The immunofluorescence images in fig 3C-E show a group of five intralobular ducts, probably close to where they converge and emerge from the hilus of the lobule. All show AQPI labelling of the apical and basolateral membranes (fig 3C). CFTR labelling of the apical plasma membrane domains (fig 3D) is much weaker here than in intercalated ducts that are visible elsewhere in the section.

Interlobular ducts, which are larger and located within the connective tissue septa between the lobules, showed much weaker AQP1 labelling (fig 3F-H). In the small and medium sized interlobular ducts, AQPI was observed at the apical membrane but rarely at the basolateral membrane (fig 3F, $3 \mathrm{G}$ ). In the larger interlobular ducts (fig $3 \mathrm{H}$ ), there was no evidence of any AQPl expression in the ductal epithelium.

\section{AQP1 distribution in rat and mouse pancreas}

In a previous study of aquaporin expression in rat pancreas, ${ }^{12}$ we failed to detect any AQPI expression in ductal cells despite strong labelling of endothelial cells in capillaries and other blood vessels. However, by raising the concentration of the AQPl antibody, we have now been able to detect weak labelling of interlobular ducts (fig 3J). Within the lobules of the gland however AQPI expression could only be detected in the blood vessel endothelia and appeared to be totally absent from acini and intralobular ducts (fig 3I). A similar pattern was observed in the mouse pancreas (data not shown).

\section{Immunoelectron microscopy of AQP1 localisation in human pancreas}

Figure $4 \mathrm{~A}$ shows part of an intercalated duct which has been subjected to immunoelectron microscopy with an anti-AQP1 primary antibody. The apical regions of two adjacent cells are shown at higher magnification in fig $4 \mathrm{~B}$ where dense labelling with gold particles is clearly associated with the microvilli at the apical membrane of intercalated duct cells. Figure 4C shows clear labelling of AQP1 in the basal and lateral plasma membrane domains.

\section{Immunohistochemical localisation of AQP5}

Immunoperoxidase labelling revealed that AQP5 is confined to the apical plasma membrane of intercalated duct cells (fig 5). Centroacinar cells did not exhibit any AQP5 immunolabelling. Although the very narrow lumen of intercalated ducts is only occasionally seen in longitudinal section, fig $5 \mathrm{~A}$ shows the converging intercalated ducts from two acini with strong apical membrane labelling of AQP5. In the same section, a group of acinar cells and intercalated duct cells, sharing a common lumen, is seen in transverse section, as is another narrow intercalated duct. Both of these structures show dense apical AQP5 labelling. More examples of labelling in the intercalated ducts are shown in fig 5B. AQP5 labelling was also observed at the apical membrane of intralobular ducts (fig 5I) but was totally absent from interlobular ducts (fig 5J).

\section{Colocalisation of AQP5 and CFTR in intercalated ducts}

To compare the distributions of AQP5 and CFTR, we again used the double label immunofluorescence technique. AQP5 labelling was confined to the apical plasma membrane in intercalated duct cells (fig 5C) where it was colocalised with CFTR (fig 5D, 5E). In addition, some CFTR labelling at the centre of the acini was found not to be associated with AQP5 

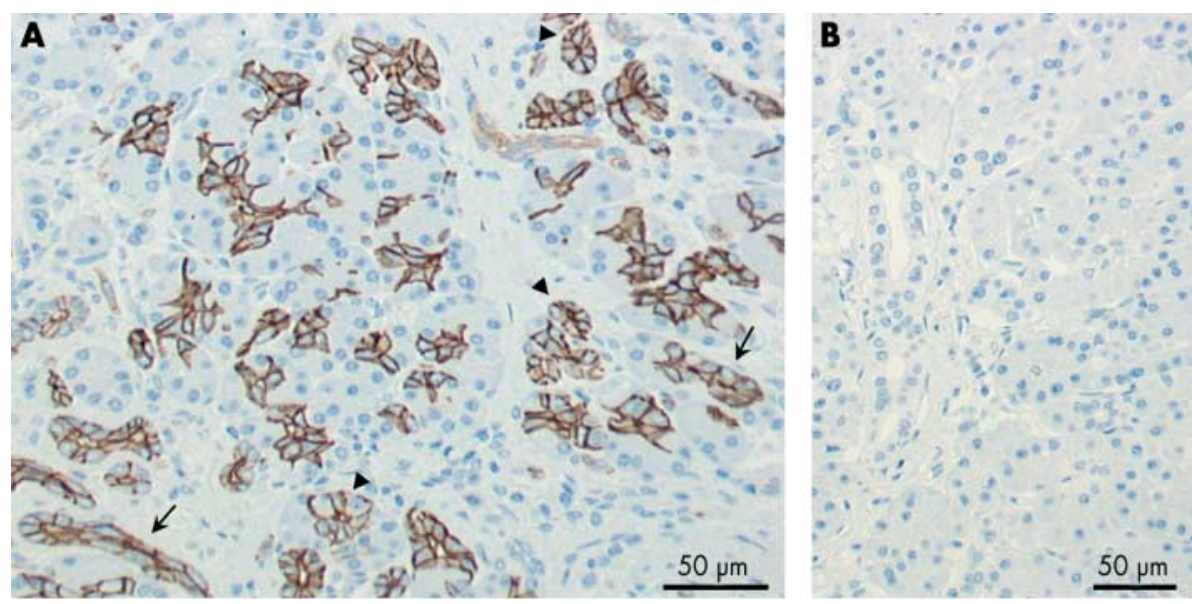

Figure 2 Immunolocalisation of aquaporin 1 (AQP1) expression in centroacinar cells and intercalated ducts of human pancreas. (A-G) Immunoperoxidase labelling of human pancreas with an affinity purified antibody to AQP1 $(2 \mu \mathrm{m}$ paraffin sections). (A) Low magnification survey field showing scattered clusters of labelled cells, some clearly identifiable as intercalated or intralobular ducts in transverse (arrowheads) or longitudinal (arrows) section. (B) Similar section exposed to primary antibody pre-adsorbed with the immunising peptide. (C-E) Labelling of centroacinar (ca) cells. Some extend to the perimeter of the acinus (arrows), between neighbouring acinar cells, and show strong
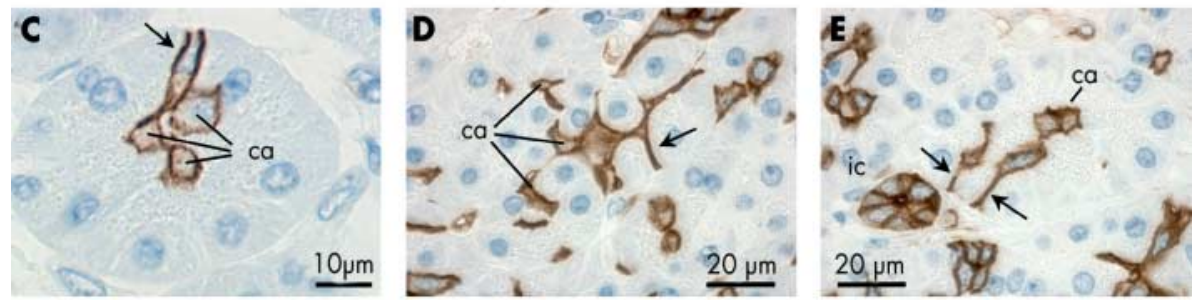

labelling of their lateral surfaces.

Others show an extensive branching morphology with interdigitations

between several acinar cells (D).

(F, G) Apical and basolateral labelling of intercalated duct (ic) cells in transverse $(F)$ and longitudinal $(G)$ section. Intercalated ducts are identifiable by their small diameter $(<20 \mu \mathrm{m})$, the small number of surrounding cells (typically 3-7 nuclei in transverse section), and very small luminal space. In (F), a chain of AQP1 labelled centroacinar cells extends along the lumen of a tubular acinus. (H-J) Immunofluorescence labelling of a paraffin section of normal human pancreas showing apical and basolateral labelling of intercalated duct cells with AQP 1 antibody $(\mathrm{H}$, green), apical labelling with cystic fibrosis transmembrane conductance regulator (CFTR) antibody (I, red), and superimposed (J) showing colocalisation (yellow) of $A Q P 1$ and CFTR at the apical membrane. Because of the very small luminal diameter of the intercalated ducts, CFTR labelling is observed at isolated points of fluorescence in ducts cut transversely and only occasionally in longitudinal profiles of the duct lumen.
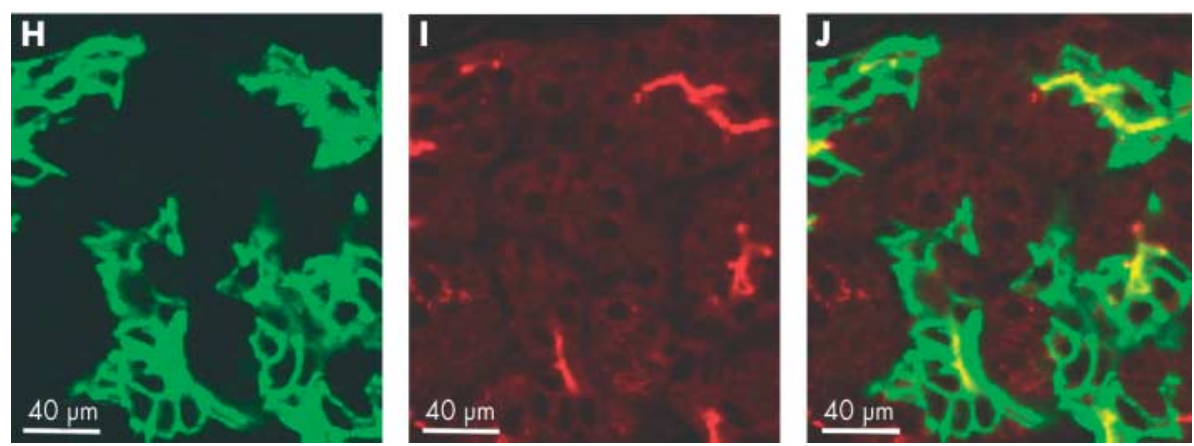

fluorescence. This is also evident in fig 5F which shows an intercalated duct leading into a single acinus. It is clear that CFTR labelling extends further into the centre of the acinus than AQP5 labelling.

\section{AQP5 in mucoid ducts and glands}

Mucoid glands were also observed occasionally in sections of human pancreas (fig 6A). Their secretory cells have flattened basally located nuclei and an enlarged apical region. As shown in fig 6A, the luminal membrane was labelled strongly with the AQP5 antibody. No AQP1 labelling was observed in these cells in a contiguous section (fig 6B). A few larger ducts lined with similar cells also showed distinct apical AQP5 labelling (fig 6C) and no AQP1 (fig 6D).

\section{Other aquaporins}

Despite the positive RT-PCR results for AQP3 and AQP4, we were unable to confirm expression of these isoforms by immunohistochemistry. An affinity purified antibody for AQP3, which gave positive results in human salivary glands as described previously, ${ }^{24}$ failed to produce plasma membrane labelling in human pancreas (not shown). Moreover, no labelling was detected with antibodies to rat or human AQP4 (not shown).

An antihuman AQP8 antibody showed distinct labelling of the apical plasma membrane domains of the acinar cells in close proximity to the zymogen granules (fig 6E). The pattern of expression was similar to what we have reported previously in rat pancreas ${ }^{12}$ and there was no obvious labelling of 

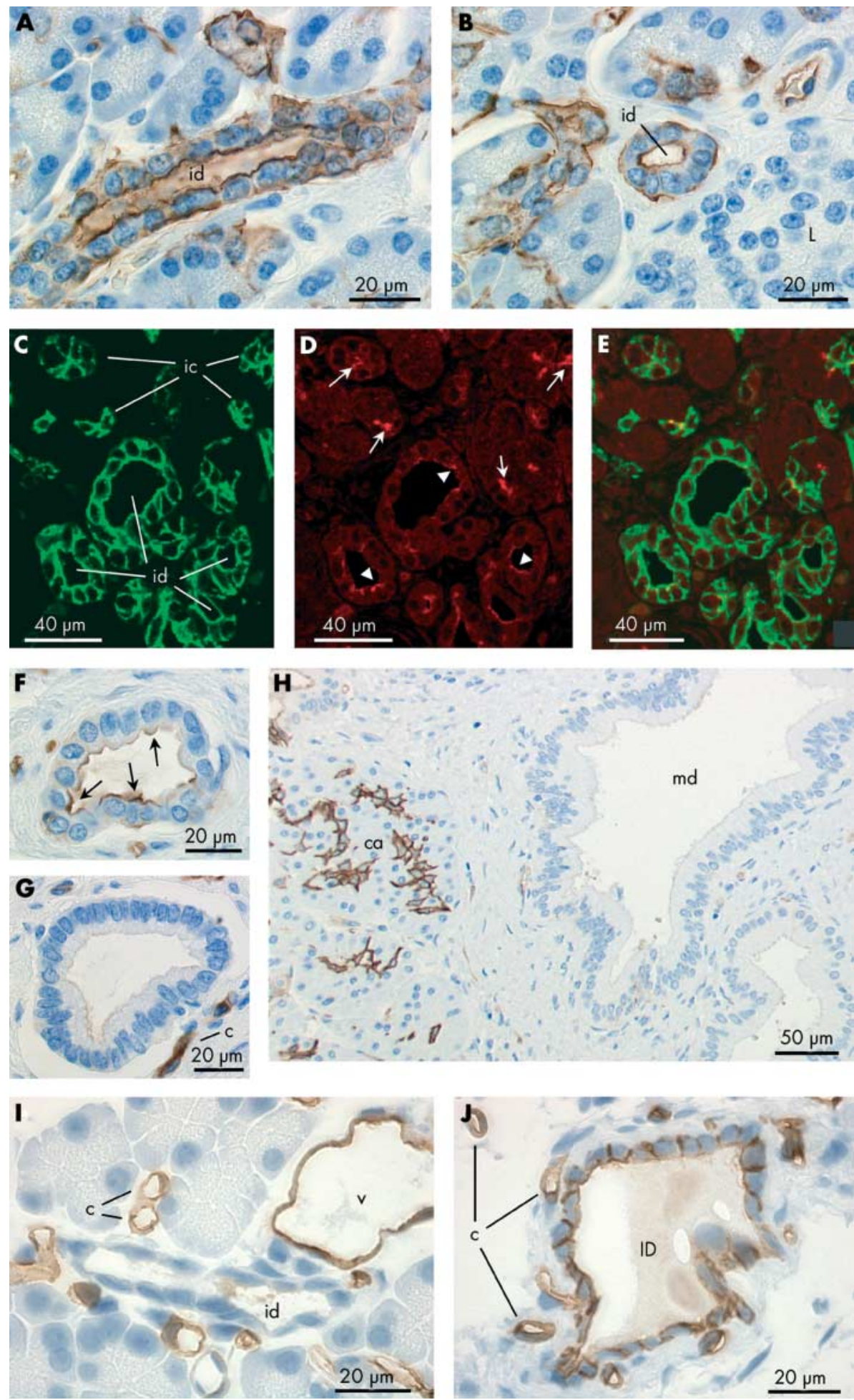

intracellular sites or of other cell types. Immunolabelling controls using non-immune IgG or non-immune serum revealed no labelling (fig 6F).

We therefore conclude that while AQP8 is expressed exclusively in acinar cells, AQP1 and AQP5 are abundantly expressed in intercalated duct cells, which are probably the main site of fluid secretion in human pancreas.

\section{DISCUSSION}

Our observations on the distribution of AQP1, -5, -8, and CFTR in the human exocrine pancreas are summarised in table 2 .
Figure 3 Immunolocalisation of aquaporin 1 (AQP1) expression in intra- and interlobular ducts of human pancreas $(\mathrm{A}-\mathrm{H})$ and rat pancreas (I-J). (A, B) Immunoperoxidase labelling of apical and basolateral membranes of intralobular ducts (id) in longitudinal (A) and transverse (B) section. Intralobular ducts are larger than intercalated ducts and have a clear luminal space surrounded by 8-14 small cuboidal cells when viewed in cross section. Note the absence of labelling from the islets of Langerhans (L) in (B). (C-E) Immunofluorescence labelling of intralobular ducts (id) with antibodies to AQP 1

(C) and cystic fibrosis transmembrane conductance regulator (CFTR) (D), and superimposed (E). While AQP labelling is uniform over the apical and basolateral surfaces (C), CFTR labelling at the apical membrane is patchy (arrowheads, D). Intercalated duct cells (ic) also show colocalisation of AQP1 and CFTR (arrows, D) at the apical membrane. (F-H) Immunoperoxidase labelling of AQP1 in interlobular and main ducts. Labelling is mainly at the apical membrane in small interlobular ducts (arrows, $F$ ) and virtually absent from the larger interlobular ducts $(G)$ and main duct (md, H). Note the strong labelling of capillaries (c, G) and centroacinar cells $(\mathrm{ca}, \mathrm{H})$ which provide a positive internal control. (I, J) Immunoperoxidase labelling of $A Q P 1$ in rat pancreas. Within the lobules (I) labelling was present in capillaries (c), venules (v), and intralobular ducts (ID). Labelling in the ductal system was confined to the interlobular ducts (id, J) and shows slightly stronger labelling at the apical and latera surfaces of the epithelial cells than at the basal surface. observed in the apical plasma membrane of intercalated duct cells and colocalised with CFTR and AQPl, strongly indicating that AQP5 also participates in pancreatic fluid production.

When stimulated with secretin, the exocrine pancreas secretes large volumes of isotonic $\mathrm{HCO}_{3}{ }^{-}$rich fluid containing relatively little enzyme activity. ${ }^{1}$ As early as 1952, Hollander

foct that AQPI is expressed in both the and basolamembrane domains of intercalated duct cells, and also in epithelial water flow. However, we found no evidence for the presence of AQP1 in acinar cells, as previously reported by presence of AQPI in acinar cells, as previously reported by 


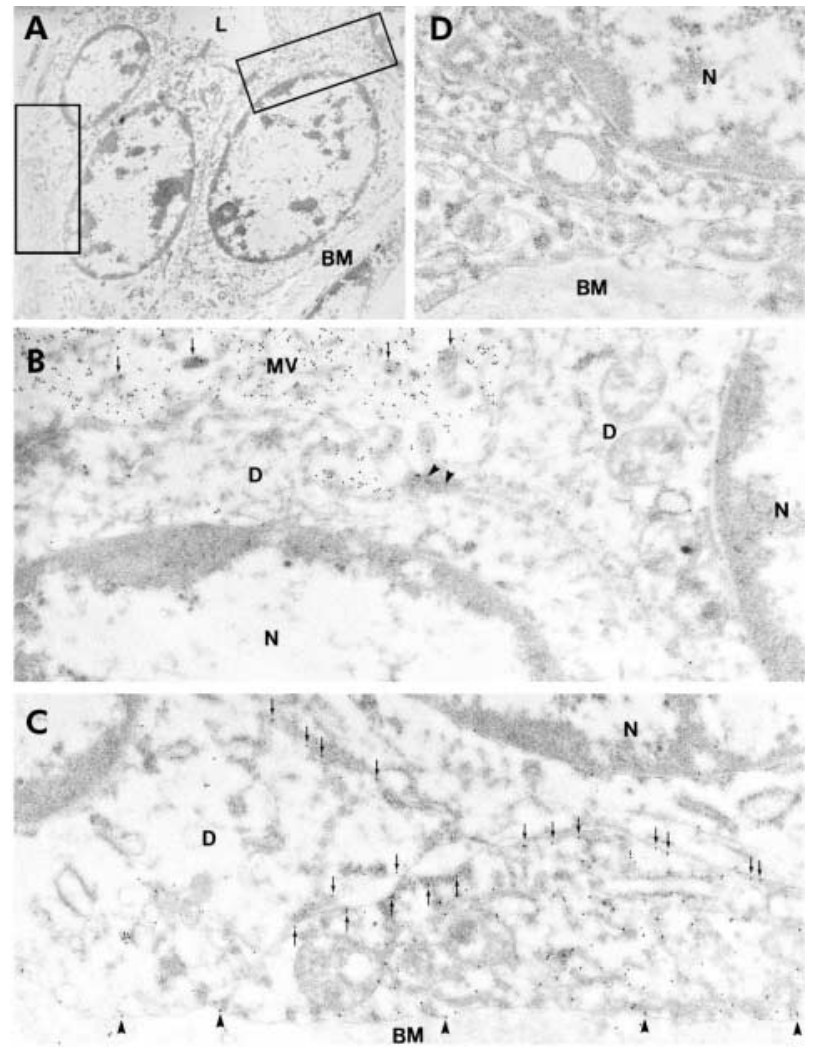

Figure 4 Immunoelectron microscopy of aquaporin 1 (AQP1) expression in intercalated duct of human pancreas. (A) Survey micrograph of an intercalated duct. Frames indicate areas magnified in (B) and (C). (B) Apical regions of two intercalated duct cells showing AQP1 labelling of microvilli (arrows) at the apical membrane. Arrowheads indicate the tight junction between adjacent duct cells. (C) Basal regions of neighbouring intercalated duct cells showing immunolabelling of AQP I at both lateral (arrows) and basal membrane domains (arrowheads). (D) Control showing absence of labelling with non-immune IgG. L, lumen; BM, basement membrane; $D$, intercalated duct cell; $N$, nucleus; $M V$, microvilli. Magnification: $A, \times 3500$; $B-D, \times 45000$.

and Birnbaum proposed that this fluid originates from "flat centro-acinar and terminal-duct cells" rather than from acinar cells. ${ }^{26}$ Subsequent studies have supported the ductal origin of the $\mathrm{HCO}_{3}^{-}$rich fluid-notably the observation that secretin evoked secretion persists in rats with severe acinar cell atrophy ${ }^{27}$ and that fluid and $\mathrm{HCO}_{3}^{-}$secretion can be evoked in short segments of interlobular duct isolated from rat and guinea pig pancreas. ${ }^{28} 29$

The aquaporins normally associated with fluid secretion in exocrine glands are AQP5 and AQP8 at the apical membrane $^{1012232430}$ and AQP3 and AQP4 at the basolateral membrane. ${ }^{1024}$ Most of these glands also express AQPl but this is generally confined to the endothelia of blood vessels. ${ }^{31}$ It is therefore perhaps surprising to find AQPI expressed apically and basolaterally in the ductal epithelial cells of the pancreas. On the other hand, its distribution in the pancreas is quite similar to that in the ductal system of the liver. Cholangiocytes, the epithelial cells that line the bile ductules, share many common features with pancreatic duct cells ${ }^{32}$ and they too express AQP1 at both the apical and basolateral membranes ${ }^{31}{ }^{33}$ and at diffuse intracellular sites. ${ }^{34}{ }^{35}$ Interestingly, Marinelli et al have suggested that AQP1 is subject to translocation from intracellular membranes to the apical plasma membrane in response to secretin. ${ }^{34}{ }^{35}$ The cytoplasmic labelling that we have observed in intercalated duct cells might indicate a similar regulated translocation of AQP1 in the pancreas.
The fact that both centroacinar cells and intercalated duct cells show labelling for AQPI supports the widely held view that centroacinar cells have a phenotype that is similar to that of intercalated duct cells. ${ }^{36-39}$ Indeed, centroacinar cells can probably be regarded as terminal intercalated duct cells which invaginate into the acinar lumen. In addition, we have shown that they may penetrate deeply, sometimes as far as the basement membrane, between adjacent acinar cells. This raises the interesting question of whether centroacinar cells have functionally distinct apical and basolateral membrane domains. ${ }^{40}$

Further down the ductal system, AQPI expression appears to decline (table 2). This suggests that while intercalated and intralobular ducts are likely to be major sites of transepithelial water flow, interlobular and main ducts act principally as conduits for conveying the secreted fluid to the duodenum. Comparing localisation of AQP1 with that of CFTR, which plays an important role in $\mathrm{HCO}_{3}^{-}$secretion, supports this hypothesis. In agreement with previous studies, ${ }^{41-43}$ we observed CFTR immunolabelling in the apical membrane of intercalated ducts but it was generally absent from interlobular ducts (table 2). Colocalisation of AQP1 and CFTR in the smaller ducts suggests that both electrolyte secretion and osmotic water flow mainly occur here. Within the acini we also detected some CFTR labelling which did not colocalise with AQP1. This indicates that CFTR is expressed at the apical membrane of acinar cells, as reported in rodents, ${ }^{44}{ }^{45}$ although previously discounted in human pancreas. ${ }^{42}{ }^{43}$

A remarkable feature of the human pancreas is coexpression of AQP1 and AQP5 at the apical membrane of intercalated duct cells. Although AQP5 immunolabelling appears relatively sparse compared with AQP1, this is partly because the lumen of intercalated ducts is extremely small, and partly because AQP5, unlike AQP1, is not expressed at the basolateral membrane, which has a much larger area. None the less, this result suggests that water transport across the very small apical surface of the intercalated ducts is facilitated by the presence of two aquaporin isoforms. Such redundancy might account for the lack of any obvious defect in pancreatic function in AQPI null humans. ${ }^{46}$

We also observed strong AQP5 labelling of mucoid glands in the human pancreas where AQP5 was clearly expressed at the apical and lateral surfaces of the cells (fig 6A). Glands of this type have been compared with Brunner's glands ${ }^{39}$ and therefore it is interesting that AQP5 expression has recently been reported at the same cellular locations in Brunner's glands of the duodenum in the rat. ${ }^{47}$

In our previous immunohistochemical study of aquaporin expression in the rat pancreas, AQPI was clearly present in blood vessels but we failed to detect it in the ductal system. ${ }^{12}$ AQPI has however recently been shown to be expressed, albeit at a low level, in interlobular ducts of the rat pancreas. ${ }^{13}{ }^{48} \mathrm{We}$ have now confirmed this, in both the rat and mouse, by using increased concentrations of AQP1 antibody (fig 3J). There is however a striking difference in the distribution of AQP1 between the human and rodent pancreas. In the rat, AQP1 is absent from centroacinar cells, intercalated ducts, and intralobular ducts but present in the small and medium sized interlobular ducts. ${ }^{48}$ In humans, AQP1 is strongly expressed in centroacinar cells, intercalated ducts, and intralobular ducts, and declines with distance along the interlobular ducts. This suggests that there is a difference in the distribution of transepithelial water flow along the pancreatic ductal systems of humans and rodents. It may of course simply reflect the different sizes of the glands and the presence of a more extensive intralobular ductal system in the larger lobules of the human pancreas.

As anticipated from previous observations in the rat, ${ }^{12}$ immunolabelling of AQP8 was observed apically in acinar cells, in close proximity to zymogen granules (fig 6E). AQP8 may therefore contribute to the production of a small amount of $\mathrm{Cl}^{-}$rich fluid that is thought to be associated with enzyme 

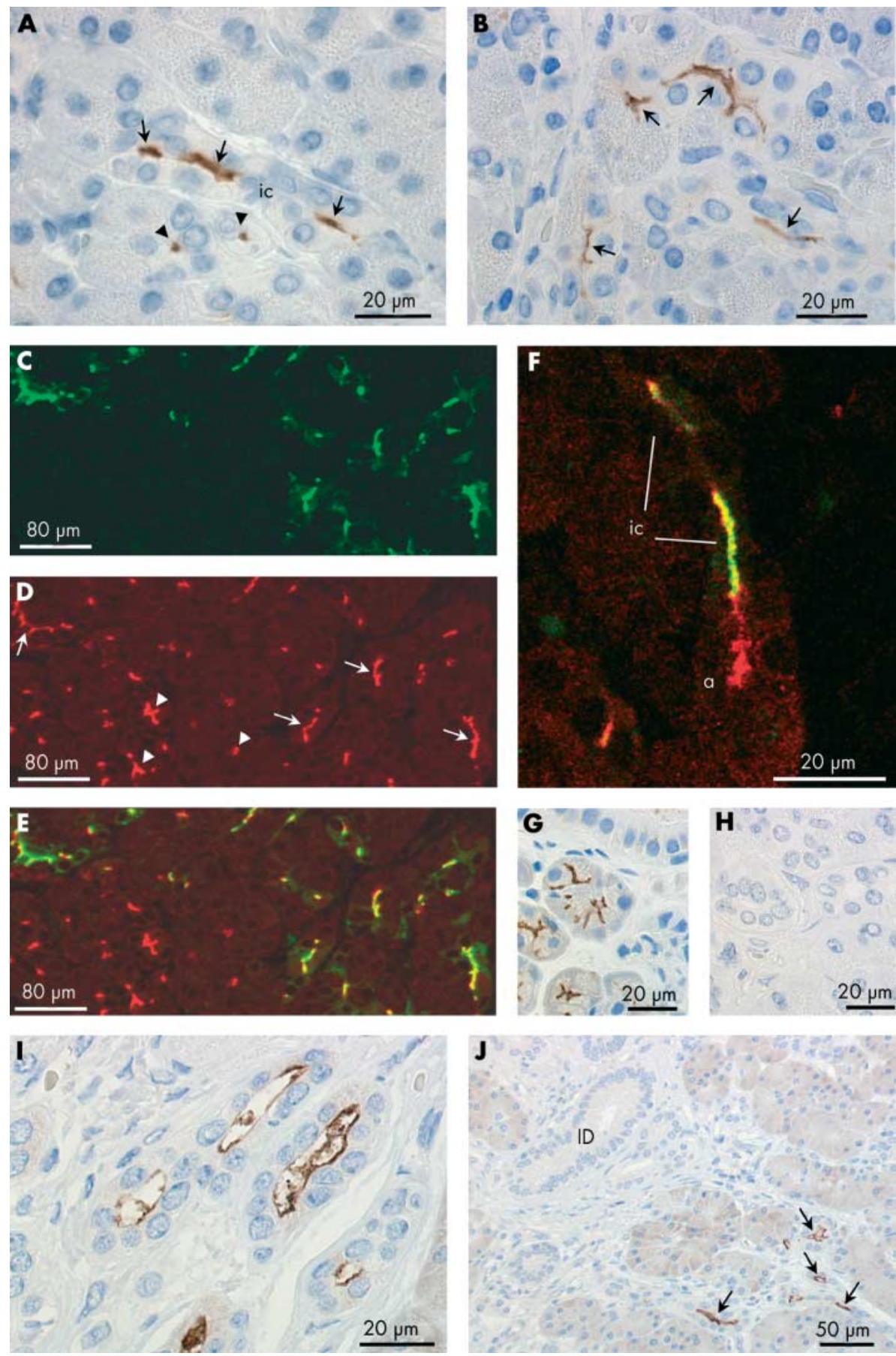

secretion. Although positive RT-PCR results were also obtained with primers for AQP3 and AQP4, neither of these isoforms was detected in normal human pancreas by immunohistochemistry. If they are present, they are presumably expressed at low levels and are therefore unlikely to participate significantly in pancreatic fluid secretion.

The two samples of pancreatic adenocarcinoma examined for AQP expression by RT-PCR resembled the normal pancreas closely (fig 1). However, only one of the two showed expression of AQP8 mRNA. As AQP8 is associated with the acinar cell phenotype, and as most tumours are of ductal origin, the absence of AQP8 from one of the samples is not surprising. It is possible that AQP8 mRNA detected in the other sample was derived from some normal acinar tissue associated with the tumour.

An interesting feature of the RT-PCR results from the pancreatic cell lines is the absence of mRNA for AQP1. Many of creatic ductal system.
Figure 5 Immunolocalisation of aquaporin 5 (AQP5) in intercalated and intralobular ducts of human pancreas. (A, B) Immunoperoxidase labelling of AQP5 at the apical membrane of intercalated ducts (ic) in longitudinal (arrows) and transverse (arrowheads) section. Both antirat and antihuman AQP5 antibodies gave the same labelling pattern. The field in $(A)$ corresponds to fig $2 G$ which shows AQP1 labelling in a contiguous section. (C) Immunofluorescence labelling of AQP5 (green) at the apical membrane of intercalated ducts. (D) Cystic fibrosis transmembrane conductance regulator (CFTR) (red) at similar locations (arrows) and also in the lumen of the acini (arrowheads). (E) Merged images showing

colocalisation of AQP5 and CFTR at the apical membrane of intercalated ducts (yellow). (F) Colocalisation of AQP5 (green) and CFTR (red) in the lumen of the intercalated duct (ic) leading from a single acinus. CFTR but not AQP5, is seen at the apical membrane of the acinar cells (a). (G) Immunoperoxidase labelling of the apical membrane and secretory canaliculi of human submandibular acinar cells confirming the specificity of the AQP5 antibody. (H) Section of human pancreas exposed to the AQP5 antibody pre-adsorbed with the immunising peptide. (I) Immunoperoxidase labelling of AQP5 at the apical membrane of intralobular duct cells in longitudinal profile. (J) Absence of AQP5 labelling in interlobular ducts (ID) despite strong labelling of intercalated and intralobular ducts (arrows).

the well differentiated "ductal" adenocarcinomas of the pancreas are thought to derive from the epithelia of the larger interlobular ducts. ${ }^{49}$ As these ducts do not express AQPl, it is perhaps not surprising that the cell lines lack this isoform. Strangely however all three cell lines examined appear to express mRNA for AQP5. In our hands, immunolabelling of AQP5 in normal human pancreas was largely confined to the intercalated ducts, which also expressed abundant AQP1. Furthermore, Capan- 1 cells have been shown to express CFTR ${ }^{50}$ which is also characteristic of intercalated and intralobular ducts rather than the large interlobular ducts. Taken together, these results suggest that the phenotypes of the cell lines do not exactly correspond to any particular segment of the pan-

In summary, the principal finding of this study was that both AQP1 and AQP5 are strongly expressed in intercalated ducts of the human exocrine pancreas. Their expression 
Table 2 Distribution of aquaporin (AQP) water channels and cystic fibrosis transmembrane conductance regulator (CFTR) in human pancreas

\begin{tabular}{|c|c|c|c|c|c|c|c|c|c|c|c|}
\hline & \multicolumn{2}{|c|}{ Acinar cells } & \multirow{2}{*}{$\begin{array}{l}\text { Centroacinar } \\
\text { cells }\end{array}$} & \multicolumn{2}{|c|}{ Intercalated ducts } & \multicolumn{2}{|c|}{ Intralobular ducts } & \multicolumn{2}{|c|}{ Small interlobular ducts } & \multicolumn{2}{|c|}{ Large interlobular ducts } \\
\hline & $\mathrm{AP}$ & $B L$ & & AP & $B L$ & AP & $B L$ & AP & BL & AP & $\mathrm{BL}$ \\
\hline CFTR & ++ & - & $?$ & ++ & - & + & - & - & - & - & - \\
\hline AQP1 & - & - & ++ & ++ & ++ & ++ & + & + & - & - & - \\
\hline AQP5 & - & - & - & ++ & - & ++ & - & - & - & - & - \\
\hline AQP8 & ++ & - & - & - & - & - & - & - & - & - & - \\
\hline
\end{tabular}

AP, apical (luminal) membrane; BL, basolateral membrane.
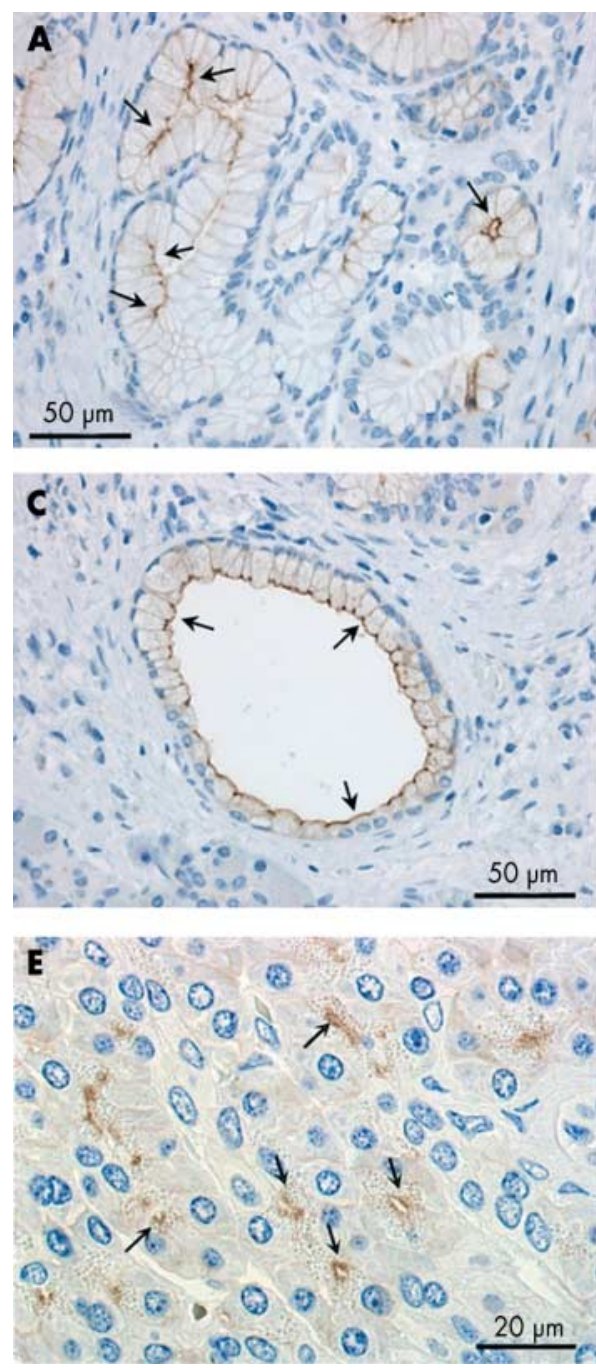
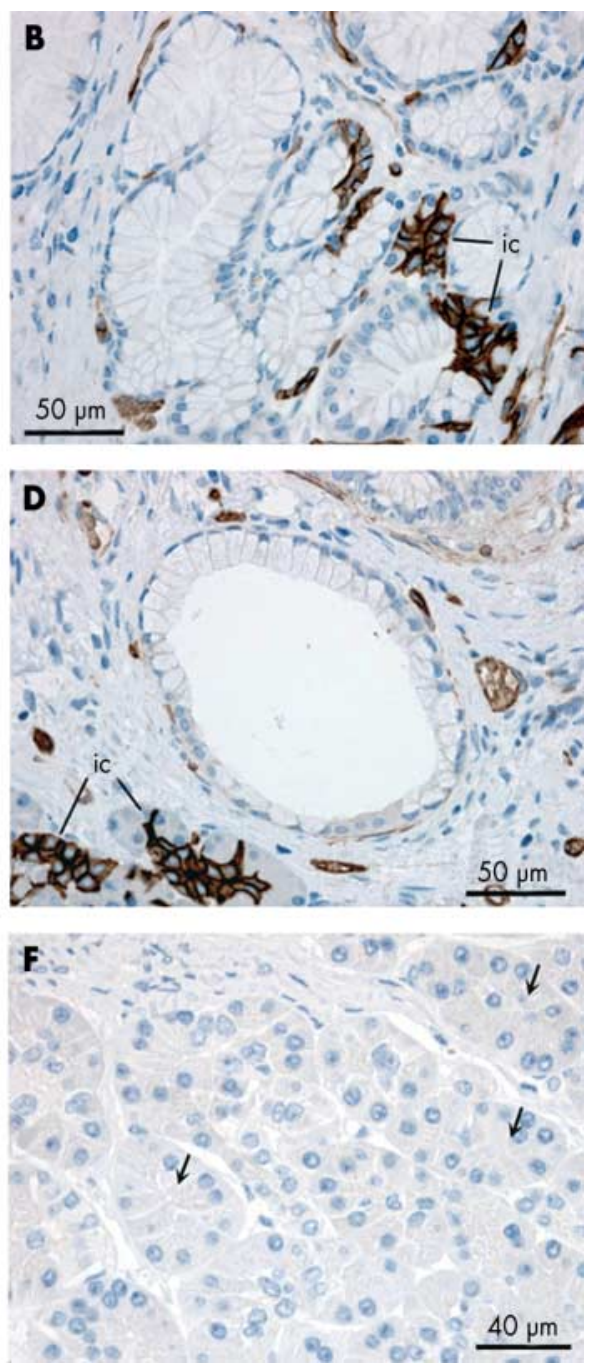

Figure 6 Immunolocalisation of aquaporin 5 (AQP5) and AQP1 in duct associated mucoid glands (A-D), AQP8 localisation in acinar cells of human pancreas (E), and immunolabelling control in human pancreas using non-immune lgG (F). (A, C) Immunoperoxidase labelling of AQP5 at the apical membrane of the glycoprotein secreting cells (arrows) of a mucoid gland $(A)$ and a large mucoid duct (C). (B, D) Absence of AQP1 labelling of mucoid cells in a contiguous section. But note the characteristic strong labelling of AQP1 in intercalated ducts (ic) associated with the mucoid gland $(B)$. The morphology of the mucoid duct epithelium $(C, D)$ was quite different from that of the columnar epithelium which is typical of interlobular ducts (fig 3G, 3H). (E) Immunoperoxidase labelling of AQP8 (arrows) at the apical pole of acinar cells in human pancreas. (F) Immunolabelling control using non-immune $\lg G$ on section of human pancreas. No labelling was observed (arrows indicate acinar cells).

correlates closely with the distribution of CFTR, a marker of ductal electrolyte secretion, and all three decline with distance downstream from the intercalated ducts. This suggests that ductal fluid secretion in the human pancreas is concentrated in the smallest terminal branches of the ductal tree and that both AQP1 and AQP5 may play a significant role in coupling the flow of water to active electrolyte secretion.

\section{ACKNOWLEDGEMENTS}

We thank Inger Merete Paulsen and Merete Pedersen for excellent technical assistance, Andi Mig for help with the RT-PCR studies, and Dr Peter Agre, Johns Hopkins University, for providing the antihuman AQP1 and AQP5 antibodies. The Water and Salt Research Center at the University of Aarhus was established and is supported by the Danish National Research Foundation (Danmarks Grundforskningsfond). This study was supported by grants from the Hungarian Ministry of
Social Welfare (ETT 2001/33), the Karen Elise Jensen Foundation, the Human Frontier Science Program, the European Commission (QLRT 2000 00778), the Novo Nordic Foundation, the Danish Medical Research Council, the University of Aarhus Research Foundation, the University of Aarhus, the Dongguk University, and the Wellcome Trust, UK.

\section{Authors' affiliations}

B Burghardt, G Varga, Molecular Oral Biology Research Group, Department of Oral Biology, Semmelweis University and Hungarian Academy of Sciences, Budapest, Hungary

G Z Rácz, Institute of Experimental Medicine, Hungarian Academy of Sciences, Budapest, Hungary

M-L Elkjaer, S Nielsen, The Water and Salt Research Centre, University of Aarhus, Denmark

T-H Kwon, Department of Physiology, Dongguk University School of 
Medicine, Korea

M C Steward, School of Biological Sciences, University of Manchester, UK

\section{REFERENCES}

1 Case RM, Argent BE. Pancreatic duct secretion: control and mechanisms of transport. In: Go VLW, DiMagno EP, Gardner JD, et al, eds. The Pancreas: Biology, Pathobiology, and Disease, 2nd edn. New York: Raven Press, 1993:301-50.

2 Case RM, Harper AA, Scratcherd T. Water and electrolyte secretion by the perfused pancreas of the cat. J Physiol 1968;196:133-49.

3 Preston GM, Agre P. Isolation of the CDNA for erythrocyte integral membrane protein of 28 kilodaltons: member of an ancient channel family. Proc Natl Acad Sci U S A 1991;88:1 111 10-14.

4 Preston GM, Carroll TP, Guggino WB, et al. Appearance of water channels in Xenopus oocytes expressing red cell CHIP28 protein. Science 1992;256:385-7.

5 Agre $\mathbf{P}$, King LS, Yasui $M$, et al. Aquaporin water channels-from atomic structure to clinical medicine. J Physiol 2002;542:3-16.

6 Borgnia M, Nielsen S, Engel A, et al. Cellular and molecular biology of the aquaporin water channels. Ann Rev Biochem 1999;68:425-58.

7 Verkman AS, Mitra AK. Structure and function of aquaporin water channels. Am J Physiol 2000;278:F13-28.

8 Nielsen S, Frokiaer J, Marples D, et al. Aquaporins in the kidney: From molecules to medicine. Physiol Rev 2002;82:205-44.

9 Ma TH, Verkman AS. Aquaporin water channels in gastrointestinal physiology. J Physiol 1999;517:317-26.

10 Nielsen S, King LS, Christensen BM, et al. Aquaporins in complex tissues. II. Subcellular distribution in respiratory and glandular tissues of rat. Amer J Physiol 1997; 273:C1549-61.

11 Koyama Y, Yamamoto T, Kondo D, et al. Molecular cloning of a new aquaporin from rat pancreas and liver. J Biol Chem 1997;272:3032933.

12 Hurley PT, Ferguson CJ, Kwon TH, et al. Expression and immunolocalization of aquaporin water channels in rat exocrine pancreas. Am J Physiol 2001;280:G701-9.

$13 \mathrm{Ko}$ SBH, Naruse S, Kitagawa $M$, et al. Aquaporins in rat pancreatic interlobular ducts. Amer J Physiol 2002;282:G324-31.

14 Raina S, Preston GM, Guggino WB, et al. Molecular-cloning and characterization of an aquaporin cDNA from salivary, lacrimal, and respiratory tissues. J Biol Chem 1995;270:1908-12.

15 Ma TH, Song YL, Gillespie A, et al. Defective secretion of saliva in transgenic mice lacking aquaporin-5 water channels. J Biol Chem 1999;274:20071-4.

16 Hasegawa $\mathbf{H}$, Lian SC, Finkbeiner WE, et al. Extrarenal tissue distribution of CHIP28 water channels by in situ hybridization and antibody staining. Am J Physiol 1994:266:C893-903.

17 Dahiya R, Kwak KS, Byrd JC, et al. Mucin synthesis and secretion in various human epithelial cancer cell lines that express the MUC-1 mucin gene. Cancer Res 1993;53:1437-43.

18 Fanjul M, Hollande E. Morphogenesis of duct-like structures in 3-dimensional cultures of human cancerous pancreatic duct cells (Capan-1). In Vitro Cell Dev Biol 1993:29A:574-84.

19 Kim YW Kern HF, Mullins TD et al Characterization of clones of a human pancreatic adenocarcinoma cell line representing different stages of differentiation. Pancreas 1989;4:353-62.

20 Burghardt B, Wenger C, Barabás K, et al. GRP-receptor-mediated signal transduction, gene expression and DNA synthesis in the human pancreatic adenocarcinoma cell line HPAF. Peptides 2001;22:1119-28.

21 Ecelbarger CA, Terris J, Frindt G, et al. Aquaporin-3 water channel localization and regulation in rat kidney. Amer J Physiol 1995:269:F663-72.

22 Terris J, Ecelbarger CA, Nielsen S, et al. Long-term regulation of four renal aquaporins in rats. Amer J Physiol 1996;271:F414-22.

23 Nejsum LN, Kwon TH, Jensen UB, et al. Functional requirement of aquaporin-5 in plasma membranes of sweat glands. Proc Natl Acad Sci US A 2002;99:511-16.

24 Gresz V, Kwon TH, Hurley PT, et al. Identification and localization of aquaporin water channels in human salivary glands. Am J Physiol 2001:281:G247-54.

25 Wallrapp C, MullerPillasch F, SolinasToldo S, et al. Characterization of a high copy number amplification at 6 q24 in pancreatic cancer identifies c-myb as a candidate oncogene. Cancer Res 1997;57:3135-9.
26 Hollander F, Birnbaum D. The role of carbonic anhydrase in pancreatic secretion. Trans NY Acad Sci 1952:15:56-8.

27 Smith PA, Sunter JP, Case RM. Progressive atrophy of pancreatic acinar tissue in rats fed a copper-deficient diet supplemented with D-penicillamine or triethylenetetramine: morphological and physiological studies. Digestion 1982;23:16-30.

28 Argent BE, Arkle S, Cullen M, et al. Morphological, biochemical and secretory studies on rat pancreatic ducts maintained in tissue culture. $Q J$ Exp Physiol 1986;71:633-48.

29 Ishiguro H, Naruse S, Steward MC, et al. Fluid secretion in interlobular ducts isolated from guinea-pig pancreas. J Physiol 1998;511:407-22.

$30 \mathrm{He} \mathrm{XJ}$, Tse CM, Donowitz M, et al. Polarized distribution of key membrane transport proteins in the rat submandibular gland. Pflugers Arch 1997:433:260-8.

31 Nielsen S, Smith BL, Christensen El, et al. Distribution of the aquaporin CHIP in secretory and resorptive epithelia and capillary endothelia. Proc Natl Acad Sci U S A 1993;90:7275-9.

32 Boyer JL. Bile-duct epithelium - frontiers in transport physiology. Am J Physiol 1996;33:G1-5.

33 Roberts SK, Yano M, Ueno Y, et al. Cholangiocytes express the aquaporin chip and transport water via a channel-mediated mechanism. Proc Natl Acad Sci U S A 1994;91:13009-13.

34 Marinelli RA, Tietz PS, Pham LD, et al. Secretin induces the apical insertion of aquaporin- 1 water channels in rat cholangiocytes. Am J Physiol 1999;276:G280-6.

35 Marinelli RA, Pham L, Agre P, et al. Secretin promotes osmotic water transport in rat cholangiocytes by increasing aquaporin- 1 water channels in plasma membrane-Evidence for a secretin-induced vesicular translocation of aquaporin-1. J Biol Chem 1997;272:12984-8.

36 Ekholm R, Edlund Y. Ultrastructure of the human exocrine pancreas. J Ultrastruct Res 1959:2:453-81.

37 Ekholm R, Zelander T, Edlund $Y$. The ultrastructural organization of the rat exocrine pancreas. II. Centroacinar cells, intercalary and intralobular ducts. J Ultrastruct Res 1962;7:73-83

38 Kodama T. A light and electron microscopic study on the pancreatic ductal system

39 Egerbacher M, Bock P. Morphology of the pancreatic duct system in mammals. Microsc Res Tech 1997;37:407-17.

40 Orci L, Perrelet A, Montesano R. Differential filipin labeling of the luminal membranes lining the pancreatic acinus. J Histochem Cytochem 1983;31:952-5.

41 Strong TV, Boehm K, Collins FS. Localization of cystic fibrosis transmembrane conductance regulator mRNA in the human gastrointestinal tract by in situ hybridization. J Clin Invest 1994;93:347-54

42 Marino CR, Matovcik LM, Gorelick FS, et al. Localization of the cystic fibrosis transmembrane conductance regulator in pancreas. J Clin Invest 1991;88:712-16.

43 Crawford I, Maloney PC, Zeitlin PL, et al. Immunocytochemical localization of the cystic fibrosis gene product CFTR. Proc Natl Acad Sci U S A 1991;88:9262-6.

44 Zeng WZ, Lee MG, Yan M, et al. Immuno and functional characterization of CFTR in submandibular and pancreatic acinar and duct cells. Am J Physiol 1997;273:C442-55.

45 Kopelman H, Ferretti E, Gauthier C, et al. Rabbit pancreatic acin express CFTR as a cAMP-activated chloride efflux pathway. Am J Physiol 1995; 38:C626-31.

46 Preston GM, Smith BL, Zeidel ML, et al. Mutations in aquaporin-1 in phenotypically normal humans without functional CHIP water channels. Science 1994:265:1585-7.

47 Parvin MN, Tsumura K, Akamatsu T, et al. Expression and localization of AQP5 in the stomach and duodenum of the rat. Biochim Biophys Acta 2002; 1542:116-24.

48 Furuya S, Naruse S, Ko SBH, et al. Distribution of aquaporin 1 in the rat pancreatic duct system examined with light- and election-microscopic immunohistochemistry. Cell Tiss Res 2002;308:75-86.

49 Adler G, Kern HF. The human exocrine pancreas in health and disease. In: Riva A, Motta PM, eds. Ultrastructure of the Extraparietal Glands of the Digestive Tract. Boston: Kluwer, 1990:1 15-46.

50 Chambers JA, Harris A. Expression of the cystic fibrosis gene and the major pancreatic mucin gene, $M U C 1$, in human ductal epithelial cells. J Cell Sci 1993;105:417-22. 